Schmerz 2016 $30: 315-316$

DOI 10.1007/s00482-016-0126-5

Online publiziert: 10. Juni 2016

(c) Deutsche Schmerzgesellschaft e.V. Published by Springer-Verlag Berlin Heidelberg - all rights reserved 2016

CrossMark

\author{
M. Schiltenwolf' $\cdot$ K. Kieselbach ${ }^{2} \cdot$ C. Bozzaro ${ }^{3}$ \\ 'Konservative Orthopädie und Schmerztherapie, Klinik für Orthopädie und Unfallchirurgie, Zentrum für \\ Orthopädie, Unfallchirurgie und Paraplegiologie, Universitätsklinikum Heidelberg, Heidelberg, \\ Deutschland \\ ${ }^{2}$ Interdisziplinäres Schmerzzentrum ISZ, Universitätsklinikum Freiburg, Freiburg, Deutschland \\ ${ }^{3}$ Institut für Ethik und Geschichte der Medizin, Albert-Ludwigs-Universität Freiburg, Freiburg, Deutschland
}

\title{
Schmerz aus anderen Perspektiven
}

tik und Therapie $\mathrm{zu}$ reduzieren (z.B. die Initiative „choosing wisely“, ABIM Foundation [1]), woraus abzuleiten wäre, dass die Medizin selbst ein Teil des Schmerzleidens bedingt.

\section{Lebensphänomen Schmerz}

Eine alternative Möglichkeit, diese Diskrepanz zu deuten, könnte darin bestehen, das Scheitern am Schmerz als ein Scheitern an einer immanenten Begebenheit des Menschseins zu verstehen. Zumindest drängt sich die Vermutung auf, dass eine Erweiterung der Perspektive auf den Schmerz im Sinne eines genuin menschlichen Widerfahrens und eine stärkere Fokussierung auf die Bedingungen eines gelingenden guten Lebens mit und trotz des Schmerzes hilfreich sein könnten.

Es sind in den letzten Jahren viele Beiträge auch von außerhalb der Medizin $\mathrm{zu}$ diesem Thema erschienen [2-4, 7], sodass ein solcher Zugang auch für die wissenschaftliche Fachzeitschrift Der Schmerz notwendig erscheint, um den Diskurs zum Lebensphänomen Schmerz $\mathrm{zu}$ begleiten und $\mathrm{zu}$ erweitern.

In diesem Themenheft soll es daher zwar um das Verständnis der Schmerzmedizin gehen, nichtmedizinische Perspektiven auf den Schmerz in der menschlichen Lebenserfahrung sollen aber vorrangig beleuchtet werden:

- Was bedeutet es, den Schmerz als anthropologische Konstante zu verstehen (Beitrag Bozzaro)?

- Wo sieht die Philosophie Funktionen des Schmerzes in der Identitäts- stiftung (Beitrag Friedrich und Tambornino)?

- Werden Anliegen von Langzeitarbeitslosen durch Ansprüche an die Rentenversicherung in die Kategorien von chronischen Schmerzen umgedeutet (Beitrag Braungardt und Schneider)?

- Sind Schmerzdeutungen hilfreich, um habituiertes Verhalten am Arbeitsplatz zu ändern (Beitrag Dresske)?

- Was macht Schmerzpatienten süchtig (Beitrag Tretter)?

- Brauchen wir zu Schmerzpatienten mit Migrationshintergrund andere Zugänge in der therapeutischen Beziehung (Beitrag Kizilhan)?

- Brauchen wir überhaupt andere Zugänge zu Patienten mit chronischen Schmerzen (Beitrag Kieselbach, Schiltenwolf und Bozzaro)?

Wir sind überzeugt, dass auch Artikel ohne die in der Medizin üblichen empirisch validierten Studienergebnisse wichtige Beiträge zum Verständnis von Schmerzen und Hilfe zur Selbstreflexion unserer Überzeugungen und Umgangsformen mit dem Schmerz liefern können. Die Lösung des angedeuteten Dilemmas von gesteigerten medizinischen Anstrengungen und oft magerem Ertrag in Hinblick auf Schmerzüberwindung bzw. -bewältigung könnte dadurch zumindest neuen Anschub jenseits biomedizinischer oder psychologischer Ansätze finden. Ein besserer Ertrag wäre vermutlich zu erwarten, wenn Einvernehmen gewonnen werden könnte, dass Schmerzen diffevon anderer Seite angemahnt, Diagnos- 
renziert und unterschiedlich gedeutet werden müssen und dass es Schmerzerfahrungen gibt, die nicht allein durch medizinisch-technische Strategien bewältigt werden können.

Aitumool

\section{Schiltenwolf}

\section{Korrespondenzadresse}

\section{Prof. Dr. M. Schiltenwolf}

Konservative Orthopädie und Schmerztherapie, Klinik für Orthopädie und Unfallchirurgie, Zentrum für Orthopädie, Unfallchirurgie und Paraplegiologie, Universitätsklinikum Heidelberg

Schlierbacher Landstraße 200 a, 69118 Heidelberg, Deutschland

Marcus.schiltenwolf@med.uni-heidelberg.de

Interessenkonflikt. M. Schiltenwolf, K. Kieselbach und C. Bozzaro geben an, dass kein Interessenkonflikt besteht.

\section{Literatur}

1. ABIM Foundation Choosing Wisely. http://www. choosingwisely.org/.Zugegriffen: 2016

2. Blume $E$, Schnalke T, Hürlimann A, Tyradellis D (Hrsg) (2007) Schmerz: Kunst und Wissenschaft. Dumont, Köln

3. Le Breton D (2002) Der Schmerz - eine Kulturgeschichte. Diaphanes, Berlin

4. Maio G, Bozzaro C, Eichinger T (2015) Leid und Schmerz. Alber, Freiburg München

5. Nguyen US, Zhang Y, Zhu Y, Niu J, Zhang B, Felson DT (2011) Increasing prevalence of knee pain and symptomatic knee osteoarthritis: survey and cohort data. Ann Intern Med 155(11):725-732

6. RaspeH (2012) Rückenschmerzen. In:RobertKochInstitut (Hrsg) Gesundheitsberichterstattung des Bundes, Bd. 53. Robert Koch-Institut, Berlin

7. Schiltenwolf M, Herzog W (Hrsg) (2011) Die Schmerzen. Königshausen und Neumann, Würzburg 